\title{
Exponentiated Nadarajah Haghighi Poisson Distribution
}

\author{
Diouma Sira KA ${ }^{1}$, George Otieno Orwa ${ }^{2} \&$ Oscar Ngesa ${ }^{3}$ \\ ${ }^{1}$ Department of Mathematics, Pan African University Institute for Basic Sciences, Technology and Innovation, Nairobi, \\ Kenya \\ ${ }^{2}$ Department of Statistics and Actuarial Science, Jomo Kenyatta University of Agriculture and Technology, Nairobi, \\ Kenya \\ ${ }^{3}$ Department of Mathematics, Statistics and Physical Sciences, Taita Taveta University, Taita Taveta, Kenya \\ Correspondence: Diouma Sira KA, Department of Mathematics, Pan African University Institute for Basic Sciences, \\ Technology and Innovation, P. O. Box 6200000200 Nairobi, Kenya. E-mail: kadioumasira@ gmail.com
}

Received: July 14, 2019 Accepted: August 2, 2019 Online Published: August 12, 2019

doi:10.5539/ijsp.v8n5p34

URL: https://doi.org/10.5539/ijsp.v8n5p34

\begin{abstract}
This paper discusses the Exponentiated Nadarajah-Haghighi Poisson distribution focusing on statistical properties such as the Quantile, Moments, Moment Generating Functions, Order statistics and Entropy. To estimate the parameters of the model, the Maximum Likelihood Estimation method is used. To demonstrate the performance of the estimators, a simulation study is carried out. A real data set from Air conditioning system is used to highlight the potential application of the distribution.
\end{abstract}

Keywords: Poisson, Exponentiated Nadarajah-Haghighi, stochastic ordering, order statistics

\section{Introduction}

Probability distributions have a pivotal role in statistical modeling. Different kinds of data arise from various disciplines including but not limited to economics, medicine, agriculture, food security, psychology. However, there is no suitable distribution for all data set, thus, the need to extend existing distributions or develop new ones (Nasiru, 2018). Modern trends focus on defining new families of distributions, by adding more parameters, that extend classical distributions and at the same time give great versatility in data modeling. Most of the generalizations to do extensions are developed due to the following reasons: a physical or statistical theoretical argument to explain the mechanism of the generated data, an appropriate model that has previously been used successfully, and a model whose empirical fit is good to the data.

Analyzing lifetime data is very important in applied sciences. Many distributions have been used to model lifetime data. Some of these include the exponential, Weibull, gamma and Rayleigh distributions and their generalizations (Gupta \& Kundu, 1999; Nadarajah \& Kotz, 2006). The features of each distribution depends on the shapes of the hazard rate function (hrf). The shape can be bathtub or unimodal and also either increase or decrease monotonically. The Exponential distribution was the first lifetime model for which statistical methods were extensively developed. Nadarajah \& Haghighi (2011) introduced an extension of the Exponential distribution as an alternative to the Gamma, Weibull and Exponentiated Exponential (EE) distributions.

In the extension, the Cumulative Distribution Function (CDF) of the Nadarajah-Haghighi (NH) distribution is given by

$$
F(x)=1-e^{1-(1+\omega x)^{\alpha}}, x>0,
$$

where $\omega>0$ and $\alpha>0$ are scale and shape parameters, respectively. The corresponding Probability Density Function (PDF) is accordingly given by

$$
f(x)=\alpha \omega(1+\omega x)^{\alpha-1} e^{1-(1+\omega x)^{\alpha}} .
$$

Exponentiated distributions can be obtained by using a positive real number $\beta$ as an index to the CDF. That is, if we have $\mathrm{CDF} F(x)$ of any random variable $X$, then the function

$$
G(x)=[F(x)]^{\beta}, \beta>0
$$

is, without loss of Generality, called an Exponentiated distribution.

The PDF is therefore given by

$$
g(x)=\frac{d G(x)}{d x}=\beta[F(x)]^{\beta-1} f(x) .
$$


Abdul-Moniem (2015) proposed the Exponentiated NH (ENH) model, whose CDF is given by

$$
F(x)=\left[1-e^{1-(1+\omega x)^{\alpha}}\right]^{\beta}, x \geq 0,
$$

where $\alpha>0$ and $\beta>0$ are shape parameters, and $\omega>0$ is the scale parameter of the distribution.

The ENH density function is given by

$$
f(x)=\alpha \omega \beta(1+\omega x)^{\alpha-1}\left[1-e^{1-(1+\omega x)^{\alpha}}\right]^{\beta-1} e^{1-(1+\omega x)^{\alpha}}
$$

Extensions of the NH distribution arise in different areas of research as discussed for instance, Vatto et al. (2016) proposed the Exponentiated Generalized NH (EGNH) distribution. Dias et al. (2016) proposed a new distribution called the beta NH (BNH) distribution. Khan et al. (2018) proposed a two-parameter weighted NH (WNH) distribution. Yousof \& Korkmaz (2017) introduced a three-parameter Topp-Leone NH (TLNH) distribution. Tahir et al. (2018) proposed the inverted NH (INH) distribution. Finally Kumar \& Kumar (2018) proposed the transmuted extended exponential distribution. In this study, another extension of the NH distribution called the Exponentiated Nadarajah-Haghighi Poisson (ENHP) distribution has been proposed by compounding the Poisson distribution with the ENH distribution. The motivation for proposing the new distribution is to provide greater flexibility and improve goodness-of-fit of modified distributions when modeling lifetime data sets.

The rest of the paper is organized as follows: In section 2, the PDF, the CDF, the Survival Function and hrf of the ENHP distribution have been defined. In section 3, statistical properties of the developed distribution have been derived. In section 4, the parameters of the distribution have been estimated using the method of Maximum Likelihood Estimation. In section 5, the Monte Carlo simulation result examine the finite sample properties of the estimators. In section 6, real data set has been used to validate the application of the model. The concluding remarks were finally given in section 7 .

\section{Exponentiated Nadarajah-Haghighi Poisson (ENHP) Distribution}

Let $M$ be the number of independent subsystems of a system functioning at a given time. Assume $M$ has zero truncated Power Series distribution with probability mass function (pmf) given by

$$
\mathbb{P}(M=m)=\frac{a_{m} \lambda^{m}}{C(\lambda)}, m=1,2, \cdots,
$$

If $a_{m}=\frac{1}{m !}$ and $C(\lambda)=e^{\lambda}-1$, then the pmf of a zero truncated Poisson distribution is given by

$$
\mathbb{P}(M=m)=\frac{\lambda^{m}}{m !\left(e^{\lambda}-1\right)}, m=1,2, \cdots, \lambda>0
$$

See (Noack, 1950). Let each subsystem failure time follow the ENH distribution (Abdul-Moniem, 2015) with CDF given by

$$
G(x)=\left[1-e^{1-(1+\omega x)^{\alpha}}\right]^{\beta}, x>0, \alpha>0, \beta>0, \omega>0 .
$$

If $T_{j}$ is the failure time of the $j^{\text {th }}$ subsystem and $X$ represents the failure time of the first out of $M$ operating subsystems such that $X=\min \left(T_{1}, T_{2}, \cdots T_{M}\right)$. Then the conditional CDF of $X$ given $M$ is

$$
\begin{aligned}
F(x \mid M=m) & =1-\mathbb{P}(X>x \mid M) \\
& =1-\mathbb{P}\left(T_{1}>x, \cdots, T_{M}>x\right) \\
& =1-\left[\mathbb{P}\left(T_{1}>x\right)\right]^{m} \\
& =1-\left[1-\mathbb{P}\left(T_{1}<x\right)\right]^{m} \\
& =1-\left[1-\left(1-e^{1-(1+\omega x)^{\alpha}}\right)^{\beta}\right]^{m} \\
F(x \mid M=m) & =1-\left[1-\left(1-e^{1-(1+\omega x)^{\alpha}}\right)^{\beta}\right]^{m} .
\end{aligned}
$$




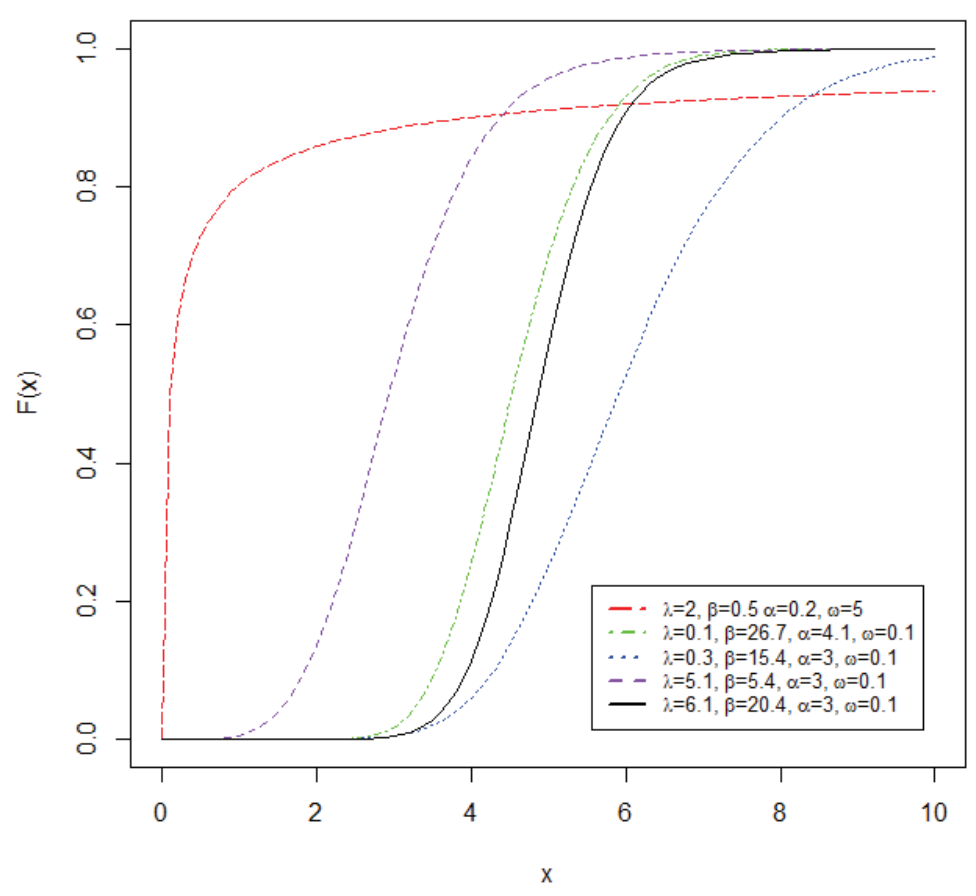

Figure 1. Plots of the ENHP CDF for some parameters values

Hence, the marginal CDF of $X$ is given by

$$
\begin{aligned}
F(x) & =\frac{1}{e^{\lambda}-1} \sum_{m=1}^{\infty} \frac{\lambda^{m}}{m !}\left\{1-\left[1-\left(1-e^{1-(1+\omega x)^{\alpha}}\right)^{\beta}\right]^{m}\right\} \\
& =\frac{1}{e^{\lambda}-1}\left\{\sum_{m=1}^{\infty} \frac{\lambda^{m}}{m !}-\sum_{m=1}^{\infty} \frac{\left[\lambda-\lambda\left(1-e^{1-(1+\omega x)^{\alpha}}\right)^{\beta}\right]^{m}}{m !}\right\} \\
& =\frac{1}{e^{\lambda}-1}\left\{e^{\lambda}-e^{\lambda-\lambda\left(1-e^{1-(1+\omega x)^{\alpha}}\right)^{\beta}}\right\} \\
& =\frac{1-e^{-\lambda\left(1-e^{1-(1+\omega x)^{\alpha}}\right)^{\beta}}}{1-e^{-\lambda}}, x>0, \\
F(x) & =\frac{1-e^{-\lambda\left(1-e^{1-(1+\omega x)^{\alpha}}\right)^{\beta}}}{1-e^{-\lambda}}, x>0,
\end{aligned}
$$

where $\alpha>0$ and $\beta>0$ are shape parameters, and $\omega>0$ and $\lambda>0$ are the scale parameters of the distribution. Figure 1 shows a monotonic non-decreasing shape bounded between 0 and 1 which is typical of any CDF.

The corresponding PDF of the ENHP distribution is obtained by differentiating the marginal CDF and is given by

$$
f(x)=\frac{\lambda \beta \alpha \omega(1+\omega x)^{\alpha-1}\left(1-e^{1-(1+\omega x)^{\alpha}}\right)^{\beta-1} e^{1-(1+\omega x)^{\alpha}} e^{-\lambda\left(1-e^{1-(1+\omega x)^{\alpha}}\right)^{\beta}}}{1-e^{-\lambda}}, x>0 .
$$

where $\alpha>0$ and $\beta>0$ are shape parameters, and $\omega>0$ and $\lambda>0$ are the scale parameters of the distribution.

Lemma 2.1. The mixture form of the PDF of the ENHP distribution can be given as

$$
f(x)=\frac{\beta}{1-e^{-\lambda}} \sum_{i=0}^{\infty} \sum_{j=0}^{\infty} \frac{(-1)^{i+j} \lambda^{i+1}}{i !(j+1)}\left(\begin{array}{c}
\beta_{i+1}-1 \\
j
\end{array}\right) e^{j+1} \alpha \omega(j+1)(1+\omega x)^{\alpha-1} e^{-(j+1)(1+\omega x)^{\alpha}},
$$

where $\beta_{i+1}=\beta(i+1)>0, \alpha>0, \beta>0, \omega>0, \lambda>0$. 


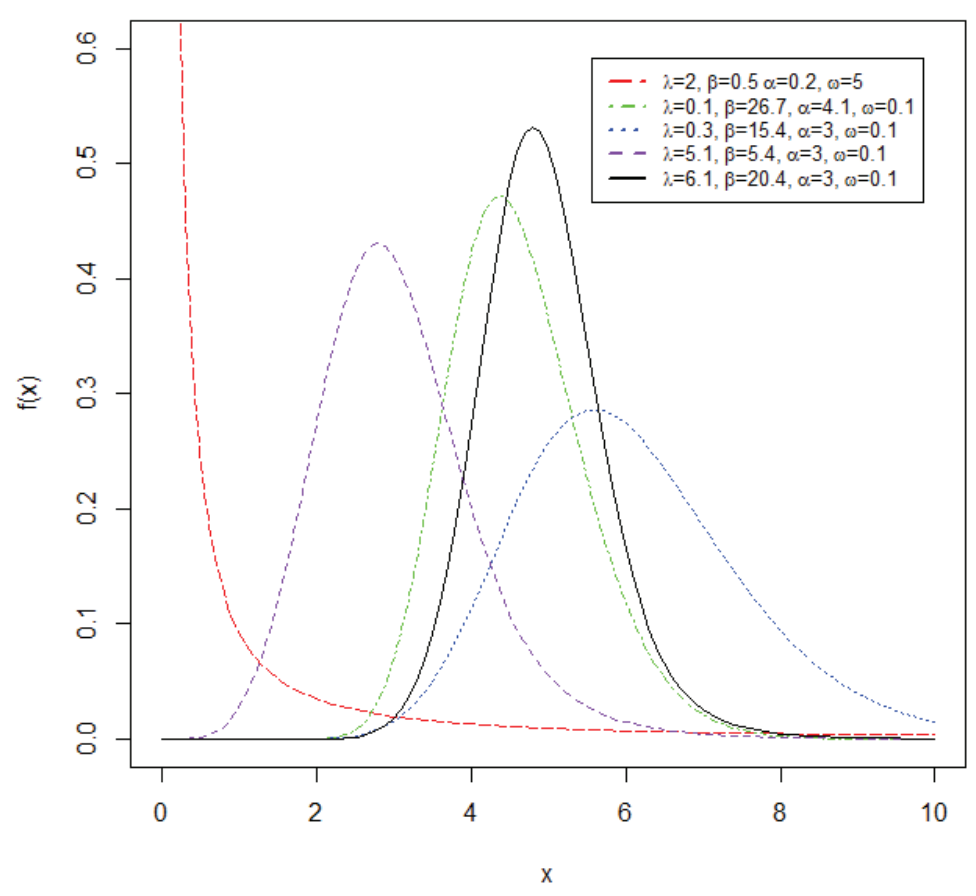

Figure 2. Plots of the ENHP PDF for some parameters values

Proof. Using the Taylor series expansion,

$$
e^{-\lambda\left(1-e^{1-(1+\omega x)^{\alpha}}\right)^{\beta}}=\sum_{i=0}^{\infty} \frac{(-1)^{i} \lambda^{i}}{i !}\left(1-e^{1-(1+\omega x)^{\alpha}}\right)^{\beta i} .
$$

Hence, the PDF of the ENHP distribution can be written as

$$
f(x)=\frac{\lambda \beta \alpha \omega(1+\omega x)^{\alpha-1} e^{1-(1+\omega x)^{\alpha}}}{1-e^{-\lambda}} \sum_{i=0}^{\infty} \frac{(-1)^{i} \lambda^{i}}{i !}\left(1-e^{1-(1+\omega x)^{\alpha}}\right)^{\beta(i+1)-1} .
$$

The following identity holds for a real non-integer $\eta$,

$$
(1-V)^{\eta-1}=\sum_{j=0}^{\infty}\left(\begin{array}{c}
\eta-1 \\
j
\end{array}\right)(-1)^{j} V^{j},|V|<1 .
$$

Using the identity in equation (16), the fact that $0<\left(1-e^{1-(1+\omega x)^{\alpha}}\right)^{\beta(i+1)-1}<1$ and $\beta_{i+1}=\beta(i+1)$, equation (15) can be expressed as

$$
f(x)=\frac{\beta}{1-e^{-\lambda}} \sum_{i=0}^{\infty} \sum_{j=0}^{\infty} \frac{(-1)^{i+j} \lambda^{i+1}}{i !}\left(\begin{array}{c}
\beta_{i+1}-1 \\
j
\end{array}\right) \alpha \omega(1+\omega x)^{\alpha-1}\left(e^{1-(1+\omega x)^{\alpha}}\right)^{j+1} .
$$

Thus,

$$
f(x)=\frac{\beta}{1-e^{-\lambda}} \sum_{i=0}^{\infty} \sum_{j=0}^{\infty} \frac{(-1)^{i+j} \lambda^{i+1}}{i !(j+1)}\left(\begin{array}{c}
\beta_{i+1}-1 \\
j
\end{array}\right) e^{j+1} \alpha \omega(j+1)(1+\omega x)^{\alpha-1} e^{-(j+1)(1+\omega x)^{\alpha}},
$$

where $\beta_{i+1}=\beta(i+1)>0, \alpha>0, \beta>0, \omega>0, \lambda>0$,

Figure 2 depicts the shapes of the PDF of the ENHP distribution for some given parameter values. It can be observed that the PDF of the ENHP can decrease or skew or symmetric, fat tail with highly flexible kurtosis, hence capable of handling variety of data from many areas like insurance and finance, survival analysis, biomedical data, reliability analysis. 
The survival function and the hrf are given by

$$
S(x)=\frac{e^{-\lambda\left(1-e^{1-(1+\omega x)^{\alpha}}\right)^{\beta}}-e^{-\lambda}}{1-e^{-\lambda}}, x>0 .
$$

and

$$
h(x)=\frac{\lambda \beta \alpha \omega(1+\omega x)^{\alpha-1}\left(1-e^{1-(1+\omega x)^{\alpha}}\right)^{\beta-1} e^{1-(1+\omega x)^{\alpha}}}{1-e^{-\lambda+\lambda\left(1-e^{1-(1+\omega x)^{\alpha}}\right)^{\beta}}}, x>0 .
$$

respectively. The plots of the hrf of the ENHP distribution, in Figure 3, show that the shapes can be monotonically decreasing, monotonically increasing, symmetrical or non-symmetrical for different parameter values. These characteristics make the ENHP distribution suitable for modeling monotonic and non-monotonic, symmetrical and non-symmetrical failure rates that are more likely to be encountered in real life situation.

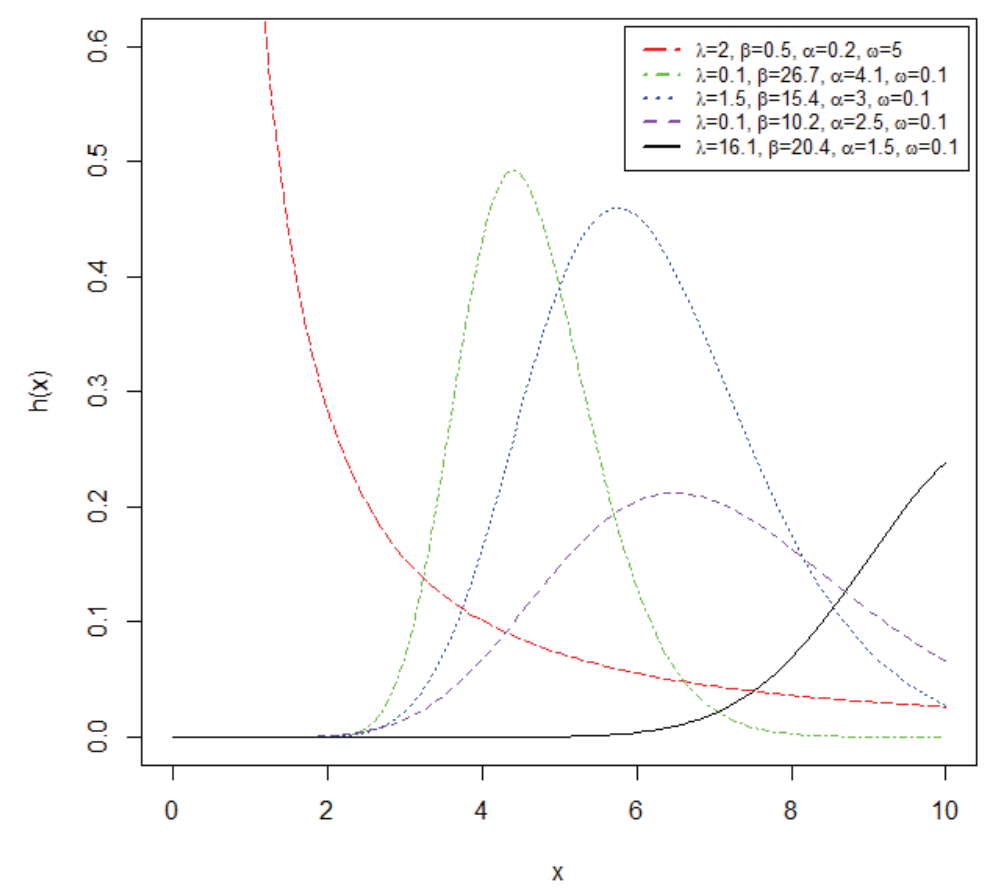

Figure 3. hrf plots for some parameters values

\section{Statistical Properties of the ENHP Distribution}

In this section various statistical properties of the ENHP distribution have been discussed.

\subsection{Quantile Function}

The quantile function is used to generate random numbers from a distribution. The quantile function of the ENHP distribution is given by

$$
Q(p)=x_{p}=\frac{1}{\omega}\left\{1-\log \left[1-\left(\log \left(1-\left(1-e^{-\lambda}\right) p\right)^{-\frac{1}{\lambda}}\right)^{\frac{1}{\beta}}\right]\right\}^{\frac{1}{\alpha}}-\frac{1}{\omega},(\alpha, \beta, \omega, \lambda>0),
$$

Substituting $p=0.25,0.5$ and 0.75 into equation (19) yields the first quartile, the median and the third quartile respectively.

\subsection{Moments}

The moments play a useful role in statistical analysis. They are used for estimating features and characteristics of a distribution such as measures of central tendency, measures of dispersion, skewness and kurtosis. 
proposition 3.1. If $X \sim \operatorname{ENHP}(\vartheta)$, where $\vartheta=\{\alpha, \beta, \omega, \lambda\}$, then the $r^{\text {th }}$ non-central moment of $X$ is given by

$$
\mu_{r}^{\prime}=\frac{\beta}{\omega^{r}\left(1-e^{-\lambda}\right)} \sum_{i=0}^{\infty} \sum_{j=0}^{\infty} \sum_{k=0}^{\infty} \frac{(-1)^{i+j+k} \lambda^{i+1}}{i !(j+1)^{\frac{r+\alpha-k}{\alpha}}} e^{j+1}\left(\begin{array}{l}
r \\
k
\end{array}\right)\left(\begin{array}{c}
\beta_{i+1}-1 \\
j
\end{array}\right) \Gamma\left(\frac{r+\alpha-k}{\alpha}, j+1\right),
$$

where $r=1,2, \cdots$ and $\Gamma(a, y)=\int_{y}^{\infty} z^{a-1} e^{-z} d z$ denotes the complementary incomplete gamma function, which is defined for all real numbers except the negative integers.

Proof. By definition, the $r^{\text {th }}$ non-central moment is given by

$$
\begin{aligned}
\mu_{r}^{\prime} & =\int_{0}^{\infty} x^{r} f(x) d x \\
& =\int_{0}^{\infty} x^{r} \frac{\beta}{1-e^{-\lambda}} \sum_{i=0}^{\infty} \sum_{j=0}^{\infty} \frac{(-1)^{i+j} \lambda^{i+1}}{i !(j+1)}\left(\begin{array}{c}
\beta_{i+1}-1 \\
j
\end{array}\right) e^{j+1} \alpha \omega(j+1)(1+\omega x)^{\alpha-1} e^{-(j+1)(1+\omega x)^{\alpha}} d x \\
& =\frac{\beta}{1-e^{-\lambda}} \sum_{i=0}^{\infty} \sum_{j=0}^{\infty} \frac{(-1)^{i+j} \lambda^{i+1}}{i !(j+1)}\left(\begin{array}{c}
\beta_{i+1}-1 \\
j
\end{array}\right) e^{j+1} \int_{0}^{\infty} x^{r} \alpha \omega(j+1)(1+\omega x)^{\alpha-1} e^{-(j+1)(1+\omega x)^{\alpha}} d x
\end{aligned}
$$

Let

$$
A_{r, j}=\int_{0}^{\infty} x^{r} \alpha \omega(j+1)(1+\omega x)^{\alpha-1} e^{-(j+1)(1+\omega x)^{\alpha}} d x \text { and } u=(j+1)(1+\omega x)^{\alpha}
$$

So

$$
d x=\frac{d u}{\alpha \omega(j+1)(1+\omega x)^{\alpha-1}} \text { and } x=\frac{1}{\omega}\left\{\left(\frac{u}{j+1}\right)^{\frac{1}{\alpha}}-1\right\} .
$$

Thus

$$
A_{r, j}=\int_{j+1}^{\infty} \frac{1}{\omega^{r}}\left\{\left(\frac{u}{j+1}\right)^{\frac{1}{\alpha}}-1\right\}^{r} e^{-u} d u
$$

The most general case of the binomial theorem is the power series identity

$$
(x+y)^{n}=\sum_{k=0}^{\infty}\left(\begin{array}{l}
n \\
k
\end{array}\right) x^{k} y^{n-k}
$$

where $\left(\begin{array}{l}n \\ k\end{array}\right)$ is a binomial coefficient and $n$ is a real number. This power series converges when $n \geq 0$ is an integer or $|x / y|<1$.

Since $\left|\left(\frac{u}{j+1}\right)^{\frac{1}{\alpha}}\right|<1$, using (22), (21) becomes

$$
A_{r, j}=\frac{1}{\omega^{r}} \sum_{k=0}^{\infty} \frac{(-1)^{k}\left(\begin{array}{l}
r \\
k
\end{array}\right)}{(j+1)^{\frac{r-k}{\alpha}}} \int_{j+1}^{\infty} u^{\frac{r-k}{\alpha}} e^{-u} d u
$$

Accordingly,

$$
\mu_{r}^{\prime}=\frac{\beta}{\omega^{r}\left(1-e^{-\lambda}\right)} \sum_{i=0}^{\infty} \sum_{j=0}^{\infty} \sum_{k=0}^{\infty} \frac{(-1)^{i+j+k} \lambda^{i+1}}{i !(j+1)^{\frac{r+\alpha-k}{\alpha}}} e^{j+1}\left(\begin{array}{l}
r \\
k
\end{array}\right)\left(\begin{array}{c}
\beta_{i+1}-1 \\
j
\end{array}\right) \Gamma\left(\frac{r+\alpha-k}{\alpha}, j+1\right)
$$

\subsection{Moment Generating Functions}

Moment Generating Functions(MGF) are special functions used to find the moments and functions of moments such as mean and variance of a random variable in a simpler way and also help in identifying which PDF or probability mass function (pmf) a random variable $X$ follows.

proposition 3.2. If $X \sim \operatorname{ENHP}(\vartheta)$, where $\vartheta=\{\alpha, \beta, \omega, \lambda\}$, then the MGF of $X$ is given by

$$
M_{X}(t)=\sum_{i=0}^{\infty} \sum_{j=0}^{\infty} \sum_{k=0}^{\infty} \sum_{r=0}^{\infty} \frac{\beta}{\omega^{r}\left(1-e^{-\lambda}\right)} \frac{(-1)^{i+j+k} t^{r} \lambda^{i+1}}{i ! r !(j+1)^{\frac{r+\alpha-k}{\alpha}}} e^{j+1}\left(\begin{array}{l}
r \\
k
\end{array}\right)\left(\begin{array}{c}
\beta_{i+1}-1 \\
j
\end{array}\right) \Gamma\left(\frac{r+\alpha-k}{\alpha}, j+1\right) .
$$


Proof: By definition, the MGF is given by

$$
M_{X}(t)=\mathbb{E}\left(e^{t X}\right)=\int_{0}^{\infty} e^{t x} f(x) d x
$$

Using the series expansion of $e^{t x}$, gives

$$
M_{X}(t)=\sum_{r=0}^{\infty} \frac{t^{r}}{r !} \int_{0}^{\infty} x^{r} f(x) d x=\sum_{r=0}^{\infty} \frac{t^{r} \mu_{r}^{\prime}}{r !}
$$

Substituting $\mu_{r}^{\prime}$ into equation (24), yields

$$
M_{X}(t)=\sum_{i=0}^{\infty} \sum_{j=0}^{\infty} \sum_{k=0}^{\infty} \sum_{r=0}^{\infty} \frac{\beta}{\omega^{r}\left(1-e^{-\lambda}\right)} \frac{(-1)^{i+j+k} t^{r} \lambda^{i+1}}{i ! r !(j+1)^{\frac{r+\alpha-k}{\alpha}}} e^{j+1}\left(\begin{array}{l}
r \\
k
\end{array}\right)\left(\begin{array}{c}
\beta_{i+1}-1 \\
j
\end{array}\right) \Gamma\left(\frac{r+\alpha-k}{\alpha}, j+1\right),
$$

which is the MGF.

\subsection{Incomplete Moment}

The incomplete moment is used to estimate the median deviation, mean deviation and measures of inequalities such as the Lorenz and Bonferroni curves.

proposition 3.3. The $r^{\text {th }}$ incomplete moment of the ENHP distribution is given by

$$
\begin{aligned}
\phi_{r}(t) & =\frac{\beta}{\omega^{r}\left(1-e^{-\lambda}\right)} \sum_{i=0}^{\infty} \sum_{j=0}^{\infty} \sum_{k=0}^{\infty} \frac{(-1)^{i+j+k} \lambda^{i+1}}{i !(j+1)^{\frac{r+\alpha-k}{\alpha}}} e^{j+1}\left(\begin{array}{l}
r \\
k
\end{array}\right)\left(\begin{array}{c}
\beta_{i+1}-1 \\
j
\end{array}\right) \\
& \times\left\{\Gamma\left(\frac{r+\alpha-k}{\alpha}, j+1\right)-\Gamma\left(\frac{r+\alpha-k}{\alpha},(j+1)(1+\omega t)^{\alpha}\right)\right\}, t>0, r=1,2, \cdots .
\end{aligned}
$$

Proof. By definition

$$
\begin{aligned}
\phi_{r}(t) & =\int_{0}^{t} x^{r} f(x) d x \\
& =\int_{0}^{t} x^{r} \frac{\beta}{1-e^{-\lambda}} \sum_{i=0}^{\infty} \sum_{j=0}^{\infty} \frac{(-1)^{i+j} \lambda^{i+1}}{i !(j+1)}\left(\begin{array}{c}
\beta_{i+1}-1 \\
j
\end{array}\right) e^{j+1} \alpha \omega(j+1)(1+\omega x)^{\alpha-1} e^{-(j+1)(1+\omega x)^{\alpha}} d x \\
& =\frac{\beta}{1-e^{-\lambda}} \sum_{i=0}^{\infty} \sum_{j=0}^{t} \frac{(-1)^{i+j} \lambda^{i+1}}{i !(j+1)}\left(\begin{array}{c}
\beta_{i+1}-1 \\
j
\end{array}\right) e^{j+1} \int_{0}^{\infty} x^{r} \alpha \omega(j+1)(1+\omega x)^{\alpha-1} e^{-(j+1)(1+\omega x)^{\alpha}} d x
\end{aligned}
$$

Let

$$
A_{r, j}=\int_{0}^{t} x^{r} \alpha \omega(j+1)(1+\omega x)^{\alpha-1} e^{-(j+1)(1+\omega x)^{\alpha}} d x \text { and } u=(j+1)(1+\omega x)^{\alpha} .
$$

So

$$
d x=\frac{d u}{\alpha \omega(j+1)(1+\omega x)^{\alpha-1}} \text { and } x=\frac{1}{\omega}\left\{\left(\frac{u}{j+1}\right)^{\frac{1}{\alpha}}-1\right\}
$$

Thus

$$
A_{r, j}=\int_{j+1}^{(j+1)(1+\omega t)^{\alpha}} \frac{1}{\omega^{r}}\left\{\left(\frac{u}{j+1}\right)^{\frac{1}{\alpha}}-1\right\}^{r} e^{-u} d u
$$

Since $\left|\left(\frac{u}{j+1}\right)^{\frac{1}{\alpha}}\right|<1$, using (22), we have

$$
\begin{aligned}
A_{r, j} & =\frac{1}{\omega^{r}} \sum_{k=0}^{\infty} \frac{(-1)^{k}\left(\begin{array}{l}
r \\
k
\end{array}\right)}{(j+1)^{\frac{r-k}{\alpha}}} \int_{j+1}^{(j+1)(1+\omega t)^{\alpha}} u^{\frac{r-k}{\alpha}} e^{-u} d u \\
& =\frac{1}{\omega^{r}} \sum_{k=0}^{\infty} \frac{(-1)^{k}\left(\begin{array}{l}
r \\
k
\end{array}\right)}{(j+1)^{\frac{r-k}{\alpha}}}\left\{\int_{j+1}^{\infty} u^{\frac{r-k}{\alpha}} e^{-u} d u-\int_{(j+1)(1+\omega t)^{\alpha}}^{\infty} u^{\frac{r-k}{\alpha}} e^{-u} d u\right\}
\end{aligned}
$$


Accordingly,

$$
\begin{aligned}
\phi_{r}(t) & =\frac{\beta}{\omega^{r}\left(1-e^{-\lambda}\right)} \sum_{i=0}^{\infty} \sum_{j=0}^{\infty} \sum_{k=0}^{\infty} \frac{(-1)^{i+j+k} \lambda^{i+1}}{i !(j+1)^{\frac{r+\alpha-k}{\alpha}}} e^{j+1}\left(\begin{array}{l}
r \\
k
\end{array}\right)\left(\begin{array}{c}
\beta_{i+1}-1 \\
j
\end{array}\right) \\
& \times\left\{\Gamma\left(\frac{r+\alpha-k}{\alpha}, j+1\right)-\Gamma\left(\frac{r+\alpha-k}{\alpha},(j+1)(1+\omega t)^{\alpha}\right)\right\} .
\end{aligned}
$$

The mean deviation, $\delta_{1}(x)$ and median deviation, $\delta_{2}(x)$, can be calculated using the relationships $\delta_{1}(x)=2 \mu F(\mu)-2 \phi_{1}(\mu)$ and $\delta_{2}(x)=\mu-2 \phi_{1}(M)$. Where $\mu=\mathbb{E}(X)$ and $M$ is the median of the ENHP random variable. $\phi_{1}(\mu)$ and $\phi_{1}(M)$ are calculated using the first incomplete moment.

\subsection{Inequality Measures}

The Bonferroni and Lorenz curves are the most widely used measures of income inequality of a given population and have various applications in economics, reliability, insurance and medicine.

proposition 3.4. The Bonferroni curve for the ENHP distribution is given by

$$
\begin{aligned}
B_{F}(t) & =\frac{\beta}{\mu \omega^{r}\left(1-e^{-\lambda\left(1-e^{1-(1+\omega)^{\alpha}}\right)^{\beta}}\right)} \sum_{i=0}^{\infty} \sum_{j=0}^{\infty} \sum_{k=0}^{\infty} \frac{(-1)^{i+j+k} \lambda^{i+1}}{i !(j+1)^{\frac{r+\alpha-k}{\alpha}}} e^{j+1}\left(\begin{array}{l}
r \\
k
\end{array}\right) \\
& \times\left(\begin{array}{c}
\beta_{i+1}-1 \\
j
\end{array}\right)\left\{\Gamma\left(\frac{r+\alpha-k}{\alpha}, j+1\right)-\Gamma\left(\frac{r+\alpha-k}{\alpha},(j+1)(1+\omega t)^{\alpha}\right)\right\} .
\end{aligned}
$$

Proof. By definition

$$
\begin{aligned}
B_{F}(t) & =\frac{1}{\mu F(t)} \int_{0}^{t} x^{r} f(x) d x \\
& =\frac{\beta}{\mu \omega^{r}\left(1-e^{-\lambda\left(1-e^{1-(1+\omega)^{\alpha}}\right)^{\beta}}\right)} \sum_{i=0}^{\infty} \sum_{j=0}^{\infty} \sum_{k=0}^{\infty} \frac{(-1)^{i+j+k} \lambda^{i+1}}{i !(j+1)^{\frac{r+\alpha-k}{\alpha}}} e^{j+1}\left(\begin{array}{l}
r \\
k
\end{array}\right)\left(\begin{array}{c}
\beta_{i+1}-1 \\
j
\end{array}\right) \\
& \times\left\{\Gamma\left(\frac{r+\alpha-k}{\alpha}, j+1\right)-\Gamma\left(\frac{r+\alpha-k}{\alpha},(j+1)(1+\omega t)^{\alpha}\right)\right\} .
\end{aligned}
$$

proposition 3.5. The Lorenz curve for the ENHP distribution is given by

$$
\begin{aligned}
L_{F}(t) & =\frac{\beta}{\mu \omega^{r}\left(1-e^{-\lambda}\right)} \sum_{i=0}^{\infty} \sum_{j=0}^{\infty} \sum_{k=0}^{\infty} \frac{(-1)^{i+j+k} \lambda^{i+1}}{i !(j+1)^{\frac{r+\alpha-k}{\alpha}}} e^{j+1}\left(\begin{array}{l}
r \\
k
\end{array}\right)\left(\begin{array}{c}
\beta_{i+1}-1 \\
j
\end{array}\right) \\
& \times\left\{\Gamma\left(\frac{r+\alpha-k}{\alpha}, j+1\right)-\Gamma\left(\frac{r+\alpha-k}{\alpha},(j+1)(1+\omega t)^{\alpha}\right)\right\} .
\end{aligned}
$$

Proof. By definition

$$
\begin{aligned}
L_{F}(t) & =\frac{1}{\mu} \int_{0}^{t} x^{r} f(x) d x \\
& =\frac{\beta}{\mu \omega^{r}\left(1-e^{-\lambda}\right)} \sum_{i=0}^{\infty} \sum_{j=0}^{\infty} \sum_{k=0}^{\infty} \frac{(-1)^{i+j+k} \lambda^{i+1}}{i !(j+1)^{\frac{r+\alpha-k}{\alpha}}} e^{j+1}\left(\begin{array}{l}
r \\
k
\end{array}\right)\left(\begin{array}{c}
\beta_{i+1}-1 \\
j
\end{array}\right) \\
& \times\left\{\Gamma\left(\frac{r+\alpha-k}{\alpha}, j+1\right)-\Gamma\left(\frac{r+\alpha-k}{\alpha},(j+1)(1+\omega t)^{\alpha}\right)\right\} .
\end{aligned}
$$

\subsection{Entropy}

Entropies are good measures of randomness and have been extensively used in information theory. Two popular entropy measures are Rényi entropy (Neyman, 1961) and Shannon entropy (Shannon, 1951). A large value of the entropy indicates a greater uncertainty in the data. The Shanon entropy is a special case of the Rényi entropy when $\eta \longrightarrow 1$ and is given by $\mathbb{E}[-\log (f(x))]$. 
proposition 3.6. If the random variable $X$ has a ENHP distribution, then the Rényi entropy of $X$ is given by

$$
E_{R}(\eta)=\frac{1}{1-\eta} \log \left\{\left(\frac{\beta \alpha \omega}{1-e^{-\lambda}}\right)^{\eta} \sum_{i=0}^{\infty} \sum_{j=0}^{\infty} \frac{(-1)^{i+j} \lambda^{\eta+i} \eta^{i} e^{\eta+j}}{i ! \alpha \omega(\eta+j)^{\frac{\eta(\alpha-1)+1}{\alpha}}}\left(\begin{array}{c}
\beta(\eta+i)-\eta \\
j
\end{array}\right) \Gamma\left(\frac{\eta(\alpha-1)-\alpha+1}{\alpha}, \eta+j\right)\right\}
$$

where $\eta>0$ and $\eta \neq 1$.

Proof. By definition,

$$
E_{R}(\eta)=\frac{1}{1-\eta} \log \left(\int_{0}^{\infty} f^{\eta}(x) d x\right), \eta>0 \text { and } \eta \neq 1
$$

From equation (12),

$$
\begin{aligned}
f^{\eta}(x) & =\left(\frac{\lambda \beta \alpha \omega}{1-e^{-\lambda}}\right)^{\eta}(1+\omega x)^{\eta(\alpha-1)}\left(1-e^{1-(1+\omega x)^{\alpha}}\right)^{\eta(\beta-1)} e^{\eta\left(1-(1+\omega x)^{\alpha}\right)} e^{-\lambda \eta\left(1-e^{1-(1+\omega x)^{\alpha}}\right)^{\beta}} \\
& =\left(\frac{\lambda \beta \alpha \omega}{1-e^{-\lambda}}\right)^{\eta}(1+\omega x)^{\eta(\alpha-1)} e^{\eta\left(1-(1+\omega x)^{\alpha}\right)} \sum_{i=0}^{\infty} \frac{(-1)^{i} \lambda^{i} \eta^{i}}{i !}\left(1-e^{1-(1+\omega x)^{\alpha}}\right)^{\beta(\eta+i)-\eta}
\end{aligned}
$$

Since $0<\left(1-e^{1-(1+\omega x)^{\alpha}}\right)^{\beta(\eta+i)-\eta}<1$, using the identity in equation (16), we have

$$
f^{\eta}(x)=\left(\frac{\beta \alpha \omega}{1-e^{-\lambda}}\right)^{\eta} \sum_{i=0}^{\infty} \sum_{j=0}^{\infty} \frac{(-1)^{i+j} \lambda^{\eta+i} \eta^{i}}{i !} e^{\eta+j}\left(\begin{array}{c}
\beta(\eta+i)-\eta \\
j
\end{array}\right)(1+\omega x)^{\eta(\alpha-1)} e^{-(\eta+j)(1+\omega x)^{\alpha}}
$$

so

$$
\int_{0}^{\infty} f^{\eta}(x) d x=\left(\frac{\beta \alpha \omega}{1-e^{-\lambda}}\right)^{\eta} \sum_{i=0}^{\infty} \sum_{j=0}^{\infty} \frac{(-1)^{i+j} \lambda^{\eta+i} \eta^{i}}{i !} e^{\eta+j}\left(\begin{array}{c}
\beta(\eta+i)-\eta \\
j
\end{array}\right) \int_{0}^{\infty}(1+\omega x)^{\eta(\alpha-1)} e^{-(\eta+j)(1+\omega x)^{\alpha}} d x
$$

Let

$$
A_{\eta}=\int_{0}^{\infty}(1+\omega x)^{\eta(\alpha-1)} e^{-(\eta+j)(1+\omega x)^{\alpha}} d x, \text { and } u=(\eta+j)(1+\omega x)^{\alpha} .
$$

so

$$
d x=\frac{d u}{\alpha \omega(\eta+j)(1+\omega x)^{\alpha-1}}, \text { and }(1+\omega x)=\left(\frac{u}{\eta+j}\right)^{\frac{1}{\alpha}} .
$$

Thus

$$
A_{\eta}=\frac{1}{\alpha \omega(\eta+j)^{\frac{\eta(\alpha-1)+1}{\alpha}}} \int_{\eta+j}^{\infty} u^{\frac{\eta(\alpha-1)-\alpha+1}{\alpha}} e^{-u} d u=\frac{\Gamma\left(\frac{\eta(\alpha-1)-\alpha+1}{\alpha}, \eta+j\right)}{\alpha \omega(\eta+j)^{\frac{\eta(\alpha-1)+1}{\alpha}}} .
$$

Therefore

$$
\int_{0}^{\infty} f^{\eta}(x) d x=\left(\frac{\beta \alpha \omega}{1-e^{-\lambda}}\right)^{\eta} \sum_{i=0}^{\infty} \sum_{j=0}^{\infty} \frac{(-1)^{i+j} \lambda^{\eta+i} \eta^{i} e^{\eta+j}}{i ! \alpha \omega(\eta+j)^{\frac{\eta(\alpha-1)+1}{\alpha}}}\left(\begin{array}{c}
\beta(\eta+i)-\eta \\
j
\end{array}\right) \Gamma\left(\frac{\eta(\alpha-1)-\alpha+1}{\alpha}, \eta+j\right) .
$$

Accordingly

$$
E_{R}(\eta)=\frac{1}{1-\eta} \log \left\{\left(\frac{\beta \alpha \omega}{1-e^{-\lambda}}\right)^{\eta} \sum_{i=0}^{\infty} \sum_{j=0}^{\infty} \frac{(-1)^{i+j} \lambda^{\eta+i} \eta^{i} e^{\eta+j}}{i ! \alpha \omega(\eta+j)^{\frac{\eta(\alpha-1)+1}{\alpha}}}\left(\begin{array}{c}
\beta(\eta+i)-\eta \\
j
\end{array}\right) \Gamma\left(\frac{\eta(\alpha-1)-\alpha+1}{\alpha}, \eta+j\right)\right\} .
$$

\subsection{Order Statistics}

The Order statistics result from transformation that involves the ordering of an entire set of observations on a random variable. They have wide applications in many areas of statistics.

Suppose $X_{1}, X_{2}, \cdots, X_{n}$ is random sample from ENHP and $X_{1: n}<X_{2: n}<\cdots<X_{n: n}$ are the corresponding order statistics. The PDF, $f_{r: n}(x)$, of $r^{\text {th }}$ order statistic $X_{r: n}$ is

$$
f_{r: n}(x)=\frac{1}{B(r, n-r+1)}[F(x)]^{r-1}[1-F(x)]^{n-r} f(x),
$$


where $F(x)$ and $f(x)$ are the CDF and PDF of the ENHP distribution respectively, and $B(.,$.$) is the beta function.$ Since $0<F(x)<1$ for $x>0$, using the binomial series expansion of $[1-F(x)]^{n-r}$, which is given by

$$
[1-F(x)]^{n-r}=\sum_{k=0}^{n-r}\left(\begin{array}{c}
n-r \\
k
\end{array}\right)(-1)^{k}[F(x)]^{k},
$$

we have

$$
f_{r: n}(x)=\frac{1}{B(r, n-r+1)} f(x) \sum_{k=0}^{n-r}\left(\begin{array}{c}
n-r \\
k
\end{array}\right)(-1)^{k}[F(x)]^{r+k-1} .
$$

Substituting the CDF and PDF of the ENHP distribution into equation (28) gives

$$
\begin{aligned}
f_{r: n}(x) & =\frac{\lambda \beta \alpha \omega(1+\omega x)^{\alpha-1}\left(1-e^{1-(1+\omega x)^{\alpha}}\right)^{\beta-1} e^{1-(1+\omega x)^{\alpha}} e^{-\lambda\left(1-e^{1-(1+\omega x)^{\alpha}}\right)^{\beta}}}{B(r, n-r+1)} \\
& \times \sum_{k=0}^{n-r}\left(\begin{array}{c}
n-r \\
k
\end{array}\right)(-1)^{k} \frac{\left(1-e^{-\lambda\left(1-e^{1-(1+\omega x)^{\alpha}}\right)^{\beta}}\right)^{r+k-1}}{\left(1-e^{-\lambda}\right)^{r+k}} .
\end{aligned}
$$

Using similar concept for expanding the density gives

$$
f_{r: n}(x)=\frac{\beta}{B(r, n-r+1)} \sum_{i=0}^{\infty} \sum_{j=0}^{\infty} \sum_{k=0}^{n-r} \sum_{l=0}^{r+k-1} \sum_{m=0}^{\infty} \sum_{s=0}^{\infty} \xi \alpha \omega(1+\omega x)^{\alpha-1} e^{-(j+s+1)(1+\omega x)^{\alpha}},
$$

where

$$
\xi=\frac{(-1)^{i+j+k+l+m+s} \lambda^{i+m+1} l^{m}}{i ! m !\left(1-e^{-\lambda}\right)^{r+k}}\left(\begin{array}{c}
\beta_{i+1}-1 \\
j
\end{array}\right)\left(\begin{array}{c}
n-r \\
k
\end{array}\right)\left(\begin{array}{c}
r+k-1 \\
l
\end{array}\right)\left(\begin{array}{c}
m \beta \\
s
\end{array}\right) e^{j+s+1}
$$

\subsection{Stochastic Ordering}

Stochastic ordering is the commonest way to show ordering mechanism in lifetime distributions.

Suppose $X_{1} \sim \operatorname{ENHP}\left(\lambda, \beta_{1}, \alpha, \omega\right)$ and $X_{2} \sim \operatorname{ENHP}\left(\lambda, \beta_{2}, \alpha, \omega\right)$, then $X_{1}$ is said to be stochastically smaller than $X_{2}$ in the

1. stochastic order $\left(X_{1} \leq_{s t} X_{2}\right)$ if the associated CDFs satisfy: $F_{X_{1}}(x) \geq F_{X_{2}}(x)$.

2. hazard rate order $\left(X_{1} \leq_{h r} X_{2}\right)$ if the associated hrfs satisfy: $h_{X_{1}}(x) \geq h_{X_{2}}(x)$.

3. likelihood ratio order $\left(X_{1} \leq_{l r} X_{2}\right)$ if the ratio of the associated PDFs given by $\frac{f_{X_{1}}(x)}{f_{X_{2}}(x)}$ decreases in $x$.

When $X_{1}$ and $X_{2}$ have a common finite left end-point support, the following implications hold

$$
X_{1} \leq_{l r} X_{2} \Longrightarrow X_{1} \leq_{h r} X_{2} \Longrightarrow X_{1} \leq_{s t} X_{2} .
$$

Suppose that the densities of $X_{1}$ and $X_{2}$ are

$$
f_{X_{1}}(x)=\frac{\lambda \beta_{1} \alpha \omega(1+\omega x)^{\alpha-1}\left(1-e^{1-(1+\omega x)^{\alpha}}\right)^{\beta_{1}-1} e^{1-(1+\omega x)^{\alpha}} e^{-\lambda\left(1-e^{1-(1+\omega x)^{\alpha}}\right)^{\beta_{1}}}}{1-e^{-\lambda}}, x>0 .
$$

and

$$
f_{X_{2}}(x)=\frac{\lambda \beta_{2} \alpha \omega(1+\omega x)^{\alpha-1}\left(1-e^{1-(1+\omega x)^{\alpha}}\right)^{\beta_{2}-1} e^{1-(1+\omega x)^{\alpha}} e^{-\lambda\left(1-e^{1-(1+\omega x)^{\alpha}}\right)^{\beta_{2}}}}{1-e^{-\lambda}}, x>0 .
$$

respectively. Then the ratio of the two densities is

$$
\frac{f_{X_{1}}(x)}{f_{X_{2}}(x)}=\frac{\beta_{1}}{\beta_{2}}\left(1-e^{1-(1+\omega x)^{\alpha}}\right)^{\beta_{1}-\beta_{2}} e^{\lambda\left[\left(1-e^{1-(1+\omega x)^{\alpha}}\right)^{\beta_{2}}-\left(1-e^{1-(1+\omega x)^{\alpha}}\right)^{\beta_{1}}\right]}
$$


Differentiating the ratio of the densities, with respect to $x$, yields

$$
\begin{aligned}
\frac{d}{d x} \frac{f_{X_{1}}(x)}{f_{X_{2}}(x)} & =A\left(\beta_{1}-\beta_{2}\right)\left(1-e^{1-(1+\omega x)^{\alpha}}\right)^{\beta_{1}-\beta_{2}-1}+A \lambda\left(1-e^{1-(1+\omega x)^{\alpha}}\right)^{\beta_{1}-\beta_{2}} \\
& \times\left\{\beta_{2}\left(1-e^{1-(1+\omega x)^{\alpha}}\right)^{\beta_{2}-1}-\beta_{1}\left(1-e^{1-(1+\omega x)^{\alpha}}\right)^{\beta_{1}-1}\right\}
\end{aligned}
$$

where

$$
A=\frac{\beta_{1}}{\beta_{2}} \alpha \omega(1+\omega x)^{\alpha-1} e^{1-(1+\omega x)^{\alpha}} e^{\lambda\left[\left(1-e^{1-(1+\omega x)^{\alpha}}\right)^{\beta_{2}}-\left(1-e^{1-(1+\omega x)^{\alpha}}\right)^{\beta_{1}}\right]}
$$

If $\beta_{2}>\beta_{1}, \frac{d}{d x} \frac{f_{X_{1}}(x)}{f_{X_{2}}(x)}<0$ which implies $\left(X_{1} \leq_{l r} X_{2}\right)$.

\section{Parameter Estimation}

In this section, the estimates of the parameters of the model is presented via method of Maximum Likelihood Estimation. Let $X_{1}, X_{2}, \cdots, X_{n}$ be a random sample of size $n$ from ENHP distribution with unknown parameter vector $\boldsymbol{\theta}=(\lambda, \beta, \alpha, \omega)^{\prime}$, then the likelihood function is defined as

$$
L\left(\boldsymbol{\theta} \mid x_{1}, x_{2}, \cdots, x_{n}\right)=\prod_{i=1}^{n} f\left(x_{i} ; \boldsymbol{\theta}\right) .
$$

Substituting from equation (12), we obtain

$$
L\left(\boldsymbol{\theta} \mid x_{1}, x_{2}, \cdots, x_{n}\right)=\prod_{i=1}^{n}\left\{\frac{\lambda \beta \alpha \omega\left(1+\omega x_{i}\right)^{\alpha-1}\left(1-e^{1-\left(1+\omega x_{i}\right)^{\alpha}}\right)^{\beta-1} e^{1-\left(1+\omega x_{i}\right)^{\alpha}} e^{-\lambda\left(1-e^{1-\left(1+\omega x_{i}\right)^{\alpha}}\right)^{\beta}}}{1-e^{-\lambda}}\right\} .
$$

The log-likelihood function for $\boldsymbol{\theta}$ is

$$
\begin{aligned}
\ell\left(\boldsymbol{\theta} \mid x_{1}, x_{2}, \cdots, x_{n}\right) & =\sum_{i=1}^{n} \log \left\{\lambda \beta \alpha \omega\left(1+\omega x_{i}\right)^{\alpha-1}\left(1-e^{1-\left(1+\omega x_{i}\right)^{\alpha}}\right)^{\beta-1}\right. \\
& \left.\times \frac{e^{1-\left(1+\omega x_{i}\right)^{\alpha}} e^{-\lambda\left(1-e^{1-\left(1+\omega x_{i}\right)^{\alpha}}\right)^{\beta}}}{1-e^{-\lambda}}\right\} \\
& =n \log (\lambda \beta \alpha \omega)-n \log \left(1-e^{-\lambda}\right)+(\alpha-1) \sum_{i=1}^{n} \log \left(1+\omega x_{i}\right) \\
& +\sum_{i=1}^{n}\left(1-\left(1+\omega x_{i}\right)^{\alpha}\right)+(\beta-1) \sum_{i=1}^{n} \log \left(1-e^{1-\left(1+\omega x_{i}\right)^{\alpha}}\right) \\
& -\lambda \sum_{i=1}^{n}\left(1-e^{1-\left(1+\omega x_{i}\right)^{\alpha}}\right)^{\beta} .
\end{aligned}
$$

The score functions are obtained by finding the partial derivatives of the log-likelihood function with respect to the parameters $\lambda, \beta, \alpha$ and $\omega$. they are defined as

$$
\begin{gathered}
\frac{\partial \ell}{\partial \lambda}=\frac{n}{\lambda}-\frac{n e^{-\lambda}}{1-e^{-\lambda}}-\sum_{i=1}^{n}\left(1-e^{1-\left(1+\omega x_{i}\right)^{\alpha}}\right)^{\beta} \\
\frac{\partial \ell}{\partial \beta}=\frac{n}{\beta}+\sum_{i=1}^{n} \log \left(1-e^{1-\left(1+\omega x_{i}\right)^{\alpha}}\right)-\lambda \sum_{i=1}^{n}\left(1-e^{1-\left(1+\omega x_{i}\right)^{\alpha}}\right)^{\beta} \log \left(1-e^{1-\left(1+\omega x_{i}\right)^{\alpha}}\right) \\
\frac{\partial \ell}{\partial \alpha}=\frac{n}{\alpha}+\sum_{i=1}^{n} \log \left(1+\omega x_{i}\right)-\sum_{i=1}^{n}\left(1+\omega x_{i}\right)^{\alpha} \log \left(1+\omega x_{i}\right) \\
+(\beta-1) \sum_{i=1}^{n} \frac{\left(1+\omega x_{i}\right)^{\alpha} \log \left(1+\omega x_{i}\right) e^{1-\left(1+\omega x_{i}\right)^{\alpha}}}{1-e^{1-\left(1+\omega x_{i}\right)^{\alpha}}} \\
-\lambda \beta \sum_{i=1}^{n}\left(1+\omega x_{i}\right)^{\alpha} \log \left(1+\omega x_{i}\right) e^{1-\left(1+\omega x_{i}\right)^{\alpha}}\left(1-e^{1-\left(1+\omega x_{i}\right)^{\alpha}}\right)^{\beta-1}
\end{gathered}
$$




$$
\begin{aligned}
\frac{\partial \ell}{\partial \omega} & =\frac{n}{\omega}+(\alpha-1) \sum_{i=1}^{n} \frac{x_{i}}{1+w x_{i}}-\alpha \sum_{i=1}^{n} x_{i}\left(1+w x_{i}\right)^{\alpha-1} \\
& -\alpha(\beta-1) \sum_{i=1}^{n} \frac{x_{i}\left(1+w x_{i}\right)^{\alpha-1} e^{1-\left(1+\omega x_{i}\right)^{\alpha}}}{1-e^{1-\left(1+\omega x_{i}\right)^{\alpha}}} \\
& -\lambda \beta \alpha \sum_{i=1}^{n} x_{i}\left(1+w x_{i}\right)^{\alpha-1} e^{1-\left(1+\omega x_{i}\right)^{\alpha}}\left(1-e^{1-\left(1+\omega x_{i}\right)^{\alpha}}\right)^{\beta-1} .
\end{aligned}
$$

Equating the score functions to zero and the system of non-linear equations solved numerically, we can get the MLE of $\boldsymbol{\theta}=(\lambda, \beta, \alpha, \omega)^{\prime}$. Thus yielding the MLE: $\hat{\boldsymbol{\theta}}=(\hat{\lambda}, \hat{\beta}, \hat{\alpha}, \hat{\omega})^{\prime}$.

\section{Monte Carlo Simulation Study}

In this section, a simulation study was carried out to investigate the Average Bias (AB) and Root Mean Square Error (RMSE) of the Maximum Likelihood Estimators for the parameters of the ENHP distribution. Various simulations are conducted for different sample sizes and different parameter values. The simulation study is repeated for $N=1000$ iterations each with sample sizes $n=50,100,200,400,600$ and parameter values in set $I: \lambda=1.5, \beta=0.4, \alpha=0.6, \omega=$ 0.3 and set $I I: \lambda=2.5, \beta=1.5, \alpha=0.8, \omega=0.5$.

The Average Bias and RMSE values of the parameters $\lambda, \beta, \alpha$ and $\omega$ for different sample sizes are presented in Table 1 . From the results, it is clear that as the sample size $n$ increases, the Average Bias, on average, decrease. It is also observed that for the parametric values, the RMSEs decrease with increasing sample size $n$.

Table 1. Monte Carlo simulation study results

\begin{tabular}{cccccc}
\hline & \multicolumn{3}{c}{ I } & \multicolumn{2}{c}{ II } \\
\hline Parameters & $\mathbf{n}$ & Average Bias & RMSE & Average Bias & RMSE \\
\hline \multirow{4}{*}{$\lambda$} & 50 & 0.6226539 & 1.664614 & -0.01643394 & 1.648036 \\
& 100 & 0.7427476 & 1.849442 & 0.1191938 & 1.743309 \\
& 200 & 0.4189384 & 1.611065 & 0.1447134 & 1.801449 \\
& 400 & 0.2047677 & 1.371155 & 0.2444762 & 1.814645 \\
& 600 & 0.1821987 & 1.278496 & 0.198225 & 1.84063 \\
& & & & & \\
$\beta$ & 50 & 0.06140861 & 0.1698807 & 0.4964533 & 2.108908 \\
& 100 & 0.02422554 & 0.0707455 & 0.2334832 & 1.244403 \\
& 200 & 0.005726971 & 0.04594976 & 0.08050179 & 0.3670992 \\
& 400 & -0.001385699 & 0.0328041 & 0.04057207 & 0.2222429 \\
& 600 & -0.002266186 & 0.02674262 & 0.03090847 & 0.1716109 \\
& & & & & \\
& 50 & 0.1998506 & 1.011527 & 0.4143147 & 1.523161 \\
& 100 & 0.2137594 & 0.6977247 & 0.241312 & 0.9864716 \\
& 200 & 0.1947008 & 0.6203456 & 0.07631328 & 0.5811534 \\
& 400 & 0.146849 & 0.4493043 & 0.0156983 & 0.2536766 \\
& 600 & 0.151142 & 0.4700457 & -0.005642115 & 0.1702314 \\
& & & & & \\
& 50 & 1.743883 & 22.12355 & 2.909462 & 16.12927 \\
$\omega$ & 100 & 0.1859374 & 0.8742861 & 1.398351 & 16.32425 \\
& 200 & 0.1140472 & 0.4876875 & 0.3537758 & 3.311303 \\
& 400 & 0.09717328 & 0.3751603 & 0.1680198 & 0.5855228 \\
& 600 & 0.083301 & 0.3346662 & 0.1328344 & 0.4441839 \\
\hline \multirow{6}{*}{$* 1$}
\end{tabular}

\section{Application on Real Dataset}

In this section, a real data set is used to illustrate the flexibility of the model in the modelling of survival data as well as compare it with competing models namely ENH (Abdul-Moniem, 2015) and TNH (Kumar \& Kumar, 2018) distributions. We fit the density functions of the ENHP, ENH and TNH distributions. The pdfs of ENH and TNH distributions are given by

$$
f_{E N H}(x)=\beta \alpha \omega(1+\omega x)^{\alpha-1}\left[1-e^{1-(1+\omega x)^{\alpha}}\right]^{\beta-1} e^{1-(1+\omega x)^{\alpha}}, x>0, \alpha>0, \omega>0, \beta>0,
$$


and

$$
f_{T N H}(x)=\alpha \omega(1+\omega x)^{\alpha-1} e^{1-(1+\omega x)^{\alpha}}\left[1-\beta+2 \beta e^{1-(1+\omega x)^{\alpha}}\right], x>0, \alpha>0, \omega>0,|\beta| \leq 1
$$

respectively.

The data set consists of the number of successive failures for the air conditioning system of each member in a fleet of 13 Boeing 720 jet airplanes as reported in Proschan (1963), and can be found in Kumar \& Kumar (2018). Table 2 displays the data set.

Table 2. Air conditioning system data

\begin{tabular}{ccccccccccccccccccc}
\hline 194 & 413 & 90 & 74 & 55 & 23 & 97 & 50 & 359 & 50 & 130 & 487 & 57 & 102 & 15 & 14 & 10 & 57 & 320 \\
261 & 51 & 44 & 9 & 254 & 493 & 33 & 18 & 209 & 41 & 58 & 60 & 48 & 56 & 87 & 11 & 102 & 12 & 5 \\
14 & 14 & 29 & 37 & 186 & 29 & 104 & 7 & 4 & 72 & 270 & 283 & 7 & 61 & 100 & 61 & 502 & 220 & 120 \\
141 & 22 & 603 & 35 & 98 & 54 & 100 & 11 & 181 & 65 & 49 & 12 & 239 & 14 & 18 & 39 & 3 & 12 & 5 \\
32 & 9 & 438 & 43 & 134 & 184 & 20 & 386 & 182 & 71 & 80 & 188 & 230 & 152 & 5 & 36 & 79 & 59 & 33 \\
246 & 1 & 79 & 3 & 27 & 201 & 84 & 27 & 156 & 21 & 16 & 88 & 130 & 14 & 118 & 44 & 15 & 42 & 106 \\
46 & 230 & 26 & 59 & 153 & 104 & 20 & 206 & 5 & 66 & 34 & 29 & 26 & 35 & 5 & 82 & 31 & 118 & 326 \\
12 & 54 & 36 & 34 & 18 & 25 & 120 & 31 & 22 & 18 & 216 & 139 & 67 & 310 & 3 & 46 & 210 & 57 & 76 \\
14 & 111 & 97 & 62 & 39 & 30 & 7 & 44 & 11 & 63 & 23 & 22 & 23 & 14 & 18 & 13 & 34 & 16 & 18 \\
130 & 90 & 163 & 208 & 1 & 24 & 70 & 16 & 101 & 52 & 208 & 95 & 62 & 11 & 191 & 14 & 71 & & \\
\hline
\end{tabular}

The maximum likelihood estimates of the parameters of ENHP, ENH and TNH distributions are given in Table 3 along with the corresponding standard errors, p-values, -2log-likelihood statistics, Akaike Information Criterion (AIC), corrected Akaike Information Criterion (AICc) and Bayesian Information Criterion (BIC).

The results based on the smaller values of the statistics:-2log likelihood, AIC, AICc, and BIC show that the ENHP distribution provides a significantly better fit than the ENH and TNH models.

Table 3. Table for MLEs of ENHP, ENH and TNH Models

\begin{tabular}{|c|c|c|c|c|c|c|c|c|}
\hline & \multicolumn{4}{|c|}{ MLEs of the parameters } & & \multicolumn{3}{|c|}{ Statistics } \\
\hline Distributions & $\lambda$ & $\beta$ & $\alpha$ & $\omega$ & $-2 \log L$ & AIC & AICc & BIC \\
\hline ENHP & 0.162542 & 1.4582876 & 0.525399 & 0.047458 & 2066.284 & 2074.284 & 2074.502 & 2087.23 \\
\hline Std. Errors & 1.586890 & 0.414505 & 0.179805 & 0.067029 & \multirow{2}{*}{\multicolumn{4}{|c|}{ 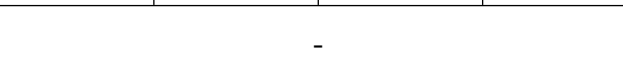 }} \\
\hline$p$-values & 0.9184169 & 0.0004346 & 0.0034774 & 0.4789303 & & & & \\
\hline ENH & \multirow{3}{*}{ - } & 0.66109706 & 3.63465741 & 0.00137937 & 2100.137 & 2106.137 & 2106.267 & 2115.846 \\
\hline Std. Errors & & 0.05468001 & 0.00133344 & 0.00009654 & \\
\hline p-values & & 0.000000 & 0.000000 & 0.000000 & & & & \\
\hline TNH & \multirow{3}{*}{ - } & 0.3280604 & 0.7892031 & 0.0134406 & 2069.668 & 2075.668 & 2075.799 & 2089.378 \\
\hline Std. Errors & & 0.4644553 & 0.1642773 & 0.0080188 & \\
\hline$p$-values & & 0.47998 & 0.000000 & 0.09371 & & & & \\
\hline
\end{tabular}

Figure 4 depicts the empirical density and the fitted densities of the distributions. The plots further indicate that the ENHP distribution is superior to ENH and TNH distributions in terms of empirical model fitting to survival data. 


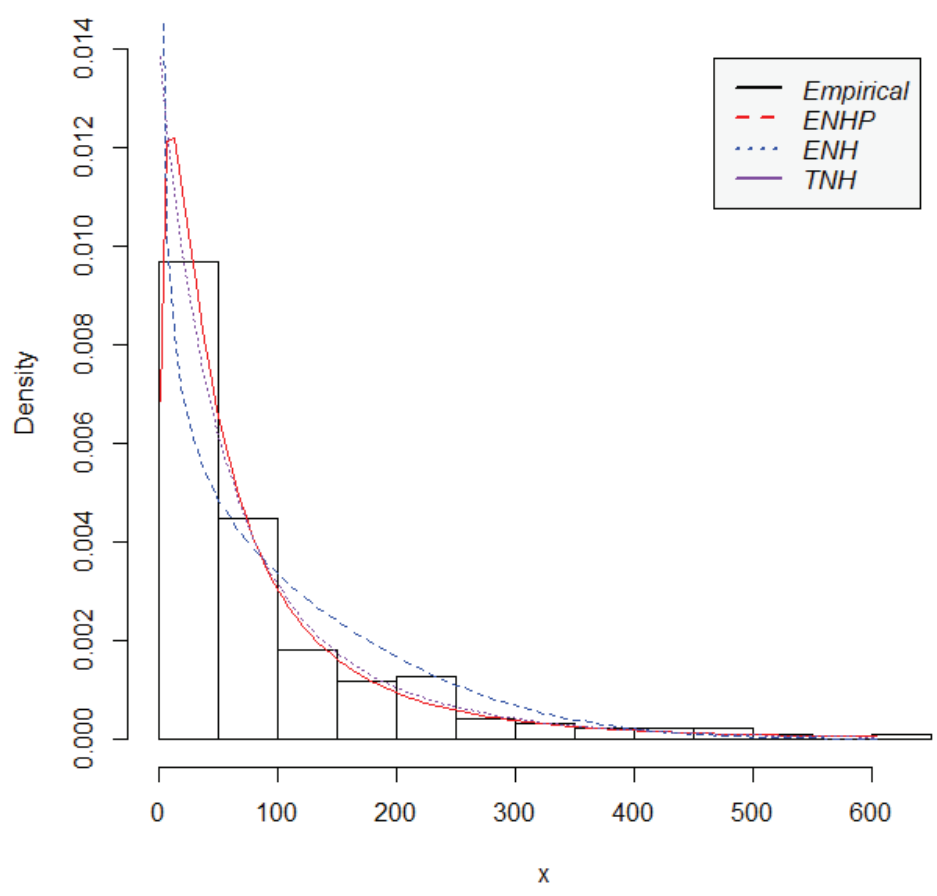

Figure 4. Empirical and fitted densities plot for Air conditioning system Data

\section{Conclusion}

In this paper, a proposal has been made on the Exponentiated Nadarajah-Haghighi Poisson distribution and studied its statistical properties. The method of Maximum Likelihood Estimation method was used to estimate the parameters of the developed distribution. Simulation studies were performed to assess the finite sample properties for the estimators of the parameters and the results showed that the estimators of parameters were stable. The application of the distribution was demonstrated using real data set and the empirical results obtained revealed the ENHP distribution is a better model compared with competing models in terms of goodness-of-fit. We recommend that further studies should be carried out by comparing the Maximum Likelihood Estimation method with the Bayesian method to compare their performance in estimating the parameters of the ENHP distribution.

\section{Acknowledgements}

The authors express their sincere thanks to the anonymous referee for a careful checking of the details and for helpful comments that improved the paper.

\section{References}

Abdul-Moniem, I. (2015). Exponentiated nadarajah and haghighi exponential distribution. International j. of Mathematical Analysis and Applications (IJMAA), 2(5), 68-73.

Dias, C. R., Alizadeh, M., \& Cordeiro, G. M. (2016). The beta nadarajah-haghighi distribution. Hacettepe University Bulletin of Natural Sciences and Engineering Series B: Mathematics and Statistics. https://doi.org/10.15672/HJMS.2017.503

Gupta, R. D., \& Kundu, D. (1999). Theory \& methods: Generalized exponential distributions. Australian E New Zealand Journal of Statistics, 4l(2), 173-188. https://doi.org/10.1111/1467-842X.00072

Khan, M. N., Saboor, A., Cordeiro, G. M., Nazir, M., \& Pescim, R. R. (2018). A weighted nadarajah and haghighi distribution.

Kumar, D., \& Kumar, M. (2018). A new generalization of the extended exponential distribution with an application. Annals of Data Science, 1-22. https://doi.org/10.1007/s40745-018-0181-0 
Nadarajah, S., \& Haghighi, F. (2011). An extension of the exponential distribution. Statistics, 45(6), 543-558. https://doi.org/10.1080/02331881003678678

Nadarajah, S., \& Kotz, S. (2006). The beta exponential distribution. Reliability engineering $\mathcal{E}$ system safety, 91(6), 689-697. https://doi.org/10.1155/2018/2931326

Nasiru, S. (2018). A New Generalization of Transformed-Transformer Family of Distributions. PhD thesis, JKUAT.

Neyman, J. (1961). Proceedings of the Fourth Berkeley Symposium on Mathematical Statistics and Probability. Univ of California Press.

Noack, A. (1950). A class of random variables with discrete distributions. The Annals of Mathematical Statistics, 21(1), 127-132. https://doi.org/10.1214/aoms/1177729894

Proschan, F. (1963). Theoretical explanation of observed decreasing failure rate. Technometrics, 5(3), 375-383. https://doi.org/10.1080/00401706.1963.10490105

Shannon, C. E. (1951). Prediction and entropy of printed english. Bell system technical journal, 30(1), 50-64. https://doi.org/10.1002/j.1538-7305.1951.tb01366.x

Tahir, M., Cordeiro, G. M., Ali, S., Dey, S., \& Manzoor, A. (2018). The inverted nadarajahChaghighi distribution: estimation methods and applications. Journal of Statistical Computation and Simulation, 1-24.

Vatto, T., Nascimento, A., WRFilho, M., Lima, M., Pinho, L., \& Cordeiro, G. (2016). Some computational and theoretical aspects of the exponentiated generalized nadarajah-haghighi distribution. arXiv preprint arXiv:1610.08876.

Yousof, H. M., \& Korkmaz, M. C. (2017). Topp-leone nadarajah-haghighi distribution. İstatistikçiler Dergisi: İstatistik ve Aktüerya, 10(2), 119-127.

\section{Copyrights}

Copyright for this article is retained by the author(s), with first publication rights granted to the journal.

This is an open-access article distributed under the terms and conditions of the Creative Commons Attribution license (http://creativecommons.org/licenses/by/4.0/). 